\title{
An evidence-based review of the potential benefits of taliglucerase alfa in the treatment of patients with Gaucher disease
}

This article was published in the following Dove Press journal:

Core Evidence

3 May 2012

Number of times this article has been viewed

\section{Carla EM Hollak}

Department of Endocrinology and Metabolism, Academic Medical Center, Amsterdam, The Netherlands
Correspondence: Carla EM Hollak Department of Endocrinology and Metabolism, F5-170, Academic Medical Center, PO Box 22660, II 00 DD Amsterdam, The Netherlands $\mathrm{Tel}+3$ I 20566607 I

Fax +3| $2069 \mid 7682$

Email c.e.hollak@amc.uva.nl
Abstract: Gaucher disease is an inherited lysosomal storage disorder, characterized by deficient activity of glucocerebrosidase leading to storage of glucocerebroside in tissue macrophages. Type I disease, the most prevalent form, lacks central nervous system involvement but presents primarily with variable degrees of hepatosplenomegaly, cytopenia, and bone disease. Intravenous enzyme replacement therapy can reverse these manifestations. In addition to the two enzymes currently authorized for use, the newest enzyme, taliglucerase alfa, is at the late stages of clinical development. Taliglucerase alfa is a unique product, as it is the first plant cell-based recombinant enzyme therapy. This review considers the existing evidence for therapeutic efficacy of taliglucerase alfa in the treatment of the non-neuronopathic manifestations of Gaucher disease. Clinical studies encompass one phase I trial in healthy volunteers, one phase III trial, and preliminary results from both an extension study and a switch study. In the 9-month, randomized, double-blind phase III trial, treatment-naïve patients with type I Gaucher disease were treated with either 30 or $60 \mathrm{U} / \mathrm{kg}$ every 2 weeks. Dose-dependent improvements were achieved after 6 and 9 months of therapy, with reductions in spleen and liver volumes and improvements in hemoglobin levels. Platelet counts improved initially only in the higherdose group, but preliminary results from the extension study also show significant increases in the lower-dose group. Bone marrow involvement, as assessed by magnetic resonance imaging, improved in almost all patients. Taliglucerase alfa has shown a good safety profile, with few patients experiencing hypersensitivity reactions and developing antibodies. An additional enzyme replacement therapy for Gaucher disease would enable the treatment of more patients and would provide backup for unexpected production problems. Furthermore, it is expected that this new treatment would reduce the costs of therapy. Taliglucerase alfa is a valuable new treatment modality for the non-neuronopathic manifestations of Gaucher disease.

Keywords: lysosomal storage disorder, glucocerebrosidase deficiency, enzyme replacement therapy, plant-derived recombinant human glucocerebrosidase

\section{Introduction: scope, aims, and objectives}

Taliglucerase alfa (Uplyso ${ }^{\circledR}$; Protalix Biotherapeutics, Karmiel, Israel; Pfizer Inc, New York, NY) is a plant-derived recombinant human glucocerebrosidase. Glucocerebrosidase (EC 3.2.1.45) is an acid hydrolase that catalyzes the breakdown of glucocerebroside (or glucosylceramide) into glucose and ceramide. Deficiency of the enzymatic activity results in buildup of undegraded glucosylceramide in macrophages, which causes Gaucher disease. ${ }^{1}$ Replacement - or, more accurately, supplementation - of the deficient enzyme by intravenous administration of glucocerebrosidase, which has a modified glycan structure exposing terminal mannoses, has been shown to be safe and effective. The first enzyme preparation that clearly 
showed reversal of disease manifestations was purified from human placental tissue (Ceredase ${ }^{\circledR}$ [alglucerase]; Genzyme Corporation, Cambridge, MA). ${ }^{2}$ This enzyme preparation was later replaced by Chinese hamster ovary cell-derived recombinant glucocerebrosidase (Cerezyme ${ }^{\circledR}$ [imiglucerase]; Genzyme Corporation). Recently, another enzyme, produced in a human fibroblast cell line, has received marketing authorization $\left(\mathrm{VPRIV}^{\circledR}\right.$ [velaglucerase alfa]; Shire Human Genetic Therapies, Cambridge, MA). ${ }^{3}$ Taliglucerase alfa is the third enzyme replacement therapy for Gaucher disease and the first plant cell-derived human enzyme, and it has the potential for large-scale production at lower costs compared to the two authorized enzymes. ${ }^{4}$ So far, taliglucerase alfa has been submitted for approval to the US Food and Drug Administration and has received an orphan drug designation at the European Medicines Agency, but it has not yet received marketing authorization. This review summarizes the evidence for the use of taliglucerase alfa to treat type I Gaucher disease (Table 1).

\section{Drug properties and mechanism of action}

Taliglucerase alfa is a recombinant human glucocerebrosidase that is expressed in a plant cell-expression system (ProCellEx ${ }^{\circledR}$; Protalix Biotherapeutics). Within this system, it is possible to produce a glycoprotein closely resembling human glucocerebrosidase that can be used for enzyme replacement therapy. For the plant cell-derived glucocerebrosidase taliglucerase alfa, it has been shown that the protein already exposed terminal mannose residues, which alleviates the need for post-production processing of the glycan composition. ${ }^{5}$ Comparison of taliglucerase alfa with imiglucerase and velaglucerase alfa shows that taliglucerase alfa has two additional amino acids at the N-terminus derived from the linker used for the fusion of the signal peptide, and it has additional seven amino acids at the C-terminus derived from the vacuolar targeting signal..$^{5}$ In addition, the amino acid composition of both imiglucerase and taliglucerase alfa differs from the human beta-glucocerebrosidase at residue 495, where a mutation causes a change from arginine to histidine. Velaglucerase alfa has the same amino acid sequence as the human enzyme, but X-ray structures of all three enzymes are very similar. ${ }^{6}$ The enzymatic activity of taliglucerase alfa toward fluorescent substrate is comparable with imiglucerase, and uptake in macrophages is also similar, although the uptake of taliglucerase alfa has been reported as enhanced at higher concentrations. ${ }^{5}$ Taliglucerase alfa differs from velaglucerase alfa and imiglucerase with respect to its glycosylation, as it contains core alpha-(1,2)-xylose and core alpha-(1,3)-fucose, ${ }^{5}$ which are unique to plant-derived proteins. The number of mannose residues is higher for taliglucerase alfa than for imiglucerase; no direct comparison is made with velaglucerase alfa, which contains longer-chain high-mannose-type glycans, compared with the core mannose structures found on imiglucerase. ${ }^{6}$

\section{Drug formulation and dosing}

Taliglucerase alfa is available as a lyophilized powder for reconstitution, with an enzymatic activity of 200 units per vial. Taliglucerase alfa is administered by intravenous infusion over 1-2 hours. Investigated doses are 30-60 U/kg of body weight once every 2 weeks. ${ }^{7}$ A range of doses has been investigated in a switch study. ${ }^{8}$

Table I Core evidence clinical impact summary for biweekly intravenous administration of taliglucerase alfa during 9 months of therapy for type I Gaucher disease

\begin{tabular}{|c|c|c|}
\hline Outcome measure & Evidence & Implications \\
\hline \multicolumn{3}{|l|}{ Disease-oriented evidence } \\
\hline Hepatosplenomegaly & $\begin{array}{l}\text { Randomized clinical trial with two dose } \\
\text { regimens }^{3}\end{array}$ & $\begin{array}{l}\text { Significant decrease, indicating reversal } \\
\text { of primary clinical manifestations }\end{array}$ \\
\hline $\begin{array}{l}\text { Specific Gaucher disease } \\
\text { markers }\end{array}$ & & $\begin{array}{l}\text { Significant decrease, indicating clearance } \\
\text { of storage cells }\end{array}$ \\
\hline \multicolumn{3}{|l|}{ Patient-oriented evidence } \\
\hline $\begin{array}{l}\text { Hemoglobin levels and } \\
\text { platelet counts }\end{array}$ & $\begin{array}{l}\text { Randomized clinical trial with two dose } \\
\text { regimens }^{3}\end{array}$ & $\begin{array}{l}\text { Improvement in the majority of patients, } \\
\text { specifically in the higher-dose group }\end{array}$ \\
\hline Safety & & $\begin{array}{l}6 \% \text { infusion reactions; } 6 \% \text { non-neutralizing } \\
\text { antibodies }\end{array}$ \\
\hline \multicolumn{3}{|l|}{ Economic evidence } \\
\hline Cost-effectiveness & Unknown & $\begin{array}{l}\text { As taliglucerase alfa has not yet received a } \\
\text { marketing authorization, the price is unknown }\end{array}$ \\
\hline
\end{tabular}




\section{Clinical trials}

Pharmacokinetics: phase I and III clinical trials

Two safety studies were performed in cynomolgus monkeys. In a short, 4-week study with daily administration of taliglucerase alfa, no antibodies were detected. In a longer-term, 39-week study, with biweekly infusions, non-neutralizing antibodies could be detected in five of 24 monkeys. No infusion-related side effects were observed. ${ }^{9}$ Pharmacokinetic studies in six healthy volunteers revealed a mean half-life of 15 minutes (range: 8-32 minutes) at doses of 30 or $60 \mathrm{U} / \mathrm{kg} .{ }^{9}$ After three weekly infusions, with increasing dosages, no antibodies were detected. In the phase III study, pharmacokinetics of two doses of enzyme, 30 and $60 \mathrm{U} / \mathrm{kg}$, every 2 weeks were studied after the first infusion and after 38 weeks in 29 patients with Gaucher disease type I. Mean maximum plasma concentration, area under the plasma concentration-time curve from the time of administration to the last measured concentration, and area under the plasma concentration-time curve from the time of administration to infinity were all higher for patients in the $60 \mathrm{U} / \mathrm{kg}$ per infusion group than for those in the $30 \mathrm{U} / \mathrm{kg}$ per infusion group.? The half-life was similar to what was observed in the healthy volunteers. Two patients developed antibodies that were non-neutralizing and which did not result in adverse effects. ${ }^{7}$ No data have been published on the influence of antibody formation on the pharmacokinetic profile.

\section{Safety, tolerability, and clinical efficacy: phase III clinical trial}

The phase III clinical trial was a randomized, double-blind, parallel-group, safety and efficacy study in treatment-naïve patients with type I Gaucher disease. ${ }^{7}$ Patients eligible for the trial were required to have an intact, enlarged spleen, as the primary endpoint was the mean decrease in spleen volume. By excluding splenectomized patients, a selection is made for somewhat less severely affected patients, as splenectomy is generally associated with more advanced liver involvement and bone complications. Two dosing groups were evaluated: 30 and $60 \mathrm{U} / \mathrm{kg}$ of body weight once every 2 weeks. Secondary endpoints were a decrease in liver volume, an increase in hemoglobin and platelet levels, and a decrease in plasma chitotriosidase activity, which is a widely used biochemical marker of Gaucher disease. ${ }^{10}$ The study duration was 9 months. Of 33 randomized patients, 16 were allocated to the $30 \mathrm{U} / \mathrm{kg}$ group and 17 to the $60 \mathrm{U} / \mathrm{kg}$ group. Two patients were excluded after consent (pregnancy, withdrawal of consent) and therefore efficacy assessments were performed for 15 patients in the $30 \mathrm{U} / \mathrm{kg}$ group and 16 patients in the $60 \mathrm{U} / \mathrm{kg}$ group at 6 and 9 months. The primary endpoint, a reduction of $20 \%$ in spleen volume, was already met at 6 months. Also, significant reductions in liver volume were observed, as well as increases in hemoglobin level and platelet count. For all parameters studied, the higher dose of $60 \mathrm{U} / \mathrm{kg}$ led to a more robust response than $30 \mathrm{U} / \mathrm{kg}$ every 2 weeks. This was specifically so for platelet responses: although significant increase in platelet count was observed in the $60 \mathrm{U} / \mathrm{kg}$ group, this was not the case for patients in the $30 \mathrm{U} / \mathrm{kg}$ group. Chitotriosidase decreased rapidly in a dose-dependent manner. The most impressive improvement in hemoglobin was that achieved in patients with anemia at baseline. This was not the same for platelet response: in patients with larger spleens and lower platelet counts, platelet responses were generally less impressive. This observation has also been made in Gaucher disease patients treated with imiglucerase. ${ }^{11}$ Perhaps the differences in response between the two dose groups were related to this phenomenon, as at baseline the lower-dose group had more patients with very large spleens than the higher-dose group.

No serious adverse events were reported. Two patients developed hypersensitivity reactions: one acute reaction after $<5 \mathrm{~mL}$ of infusion, which later occurred after imiglucerase infusion as well, and one mast cell-mediated reaction. ${ }^{3}$ Adverse events that were considered to be drug related occurred in eight patients ( 28 events) and included the two patients with hypersensitivity reactions. The remaining adverse events were variable and included symptoms such as abdominal pain, headache, and pruritus, which were all mild/moderate and transient. ${ }^{?}$

\section{Additional studies}

In addition to the studies already mentioned, a number of other studies have also been performed. Results of these studies were presented recently at the Lysosomal Disease Network: WORLD Symposium 2012, held February 8-10 in San Diego, California. The first study is the extension of the phase III clinical trial. Patients could continue treatment with taliglucerase alfa, at the same dose received in the trial, for an additional 15 months. Twenty-six of the 29 patients were followed and these patients demonstrated further improvements in all parameters; no new safety concerns were reported. ${ }^{12}$ A press release from Protalix Biotherapeutics, published online on February 9, 2012, provided detailed data indicating that further spleen volume reductions from 
baseline were achieved: $54 \%$ further reduction in the $60 \mathrm{U} / \mathrm{kg}$ group and $41 \%$ further reduction in the $30 \mathrm{U} / \mathrm{kg}$ group. ${ }^{13}$ Both dosing groups also demonstrated further reductions in liver volume. Hematologic parameters improved as well: in patients with anemia at baseline, a further increase to $12.9 \mathrm{~g} / \mathrm{dL}$ was demonstrated. The prolonged period of treatment now showed that platelets increased significantly in the lower-dose group - from 65,000 to $93,000 / \mathrm{mm}^{3}$ for the $30 \mathrm{U} / \mathrm{kg}$ group - while further improvements were also observed in the $60 \mathrm{U} / \mathrm{kg}$ group. The overall positive responses were reflected by further reductions in chitotriosidase activity: by a further $76 \%$ in the $60 \mathrm{U} / \mathrm{kg}$ group and a further $61 \%$ in the $30 \mathrm{U} / \mathrm{kg}$ group. During this extension period, no serious adverse events were reported. Two patients had antibodies; the assays used for antibody testing were different from the assay used in the initial phase III trial. One patient experienced a hypersensitivity reaction after 10 months of therapy, but this patient could continue treatment with premedication.

In a separate study, bone marrow responses were evaluated in eight patients from the phase III trial. Quantitative chemical shift imaging was performed on lumbar spines to assess fat fractions. Previous studies have shown that progressive bone marrow infiltration with Gaucher cells in untreated patients results in a decrease in adipocytes and thus also a decrease in fat fractions. ${ }^{15}$ This imaging technique has been validated in Gaucher disease as an instrument to evaluate bone marrow responses to treatment, and increases in fat fractions over 12-24 months can typically be observed. Following treatment with taliglucerase alfa, early and sustained increases in bone marrow fat fractions were observed in the majority of the patients. ${ }^{16}$

Whether patients treated with imiglucerase would maintain stable disease parameters when switching to taliglucerase alfa was studied in a 9-month open-label switchover trial. ${ }^{8}$ During this trial, a severe worldwide shortage of imiglucerase (Cerezyme) occurred, which meant that many patients had their treatment with imiglucerase interrupted or were put on lower dosages. ${ }^{17}$ Therefore the protocol was amended to allow assessment of stability based upon pre-shortage historical data and to treat a larger number of patients, including children. Preliminary results for 26 adult patients were reported at the WORLD conference. ${ }^{8}$ All patients received a dose of taliglucerase alfa that was identical to their previous imiglucerase dose, ranging from 10 to $60 \mathrm{U} / \mathrm{kg}$ every 2 weeks. One patient experienced a hypersensitivity reaction. The data showed that mean hemoglobin and platelet count and liver and spleen volumes remained stable. From the aforementioned Protalix Biotherapeutics press release of
February 9, 2012, safety analysis showed that taliglucerase alfa was well tolerated, and no drug-related serious adverse events were reported. ${ }^{13}$ One patient developed IgG antibodies; these antibodies were neutralizing in an in vitro assay but negative in a cell-based assay.

\section{Summary of data on antibody formation and hypersensitivity}

No antibodies were detected in the 4-week phase I monkey study with daily administration of taliglucerase alfa. In the 39-week study, with biweekly infusions, non-neutralizing antibodies could be detected in five of 24 monkeys. No infusion-related side effects were observed. ${ }^{9}$ In the healthy volunteers, no antibodies were detected after three weekly infusions. During the phase III study, two patients developed antibodies that were non-neutralizing. New assays were developed for the extension phase of this study and during the switch study. A total of three patients from all studies in Gaucher patients tested positive for neutralizing activity in a serum assay but not in a cell-based assay. As described earlier, two of these patients were from the phase III extension trial and one was from the switch trial. ${ }^{13}$ The patients who initially tested positive with the earlier assay now tested negative with the new assays. ${ }^{14}$ In total, two patients had hypersensitivity reactions during the first part of the phase III study and two other patients had hypersensitivity reactions during the extension and the switch studies. Interestingly, hypersensitivity reactions appeared not to be related to antibody formation. The clinical impact of the presence of antibodies is currently unclear but it does not seem to affect clinical outcome.

\section{Place of taliglucerase alfa within the treatment arsenal for Gaucher disease}

Taliglucerase alfa is not yet an authorized treatment for Gaucher disease. In 2010, the European Commission granted orphan drug designation to taliglucerase alfa, and marketing applications for taliglucerase alfa have been filed in the United States, Europe, Israel, Brazil, and Australia. An important question to consider is whether there is a place for another enzyme in the treatment of Gaucher disease. Taliglucerase alfa, as well as the two enzymes currently authorized - imiglucerase and velaglucerase alfa - is incapable of crossing the blood-brain barrier and therefore it is specifically indicated for treatment of the type I manifestations of Gaucher disease - primarily, hepatosplenomegaly, 
cytopenia, and bone manifestations. Taliglucerase alfa will not offer an alternative for the neuronopathic variants of the disease. At this point it should be mentioned that there are also oral alternatives available: small molecules that act as substrate inhibitors. Miglustat (Zavesca ${ }^{\circledR}$; Actelion Pharmaceuticals Limited, Allschwil/Basel, Switzerland) is a glucose analogue that can penetrate the central nervous system and which has modest effects on Gaucher disease manifestations. However, there is no clear effect on neurological symptoms and it has been authorized for the treatment of mild to moderately severe type I Gaucher disease..$^{18} \mathrm{~A}$ new oral treatment currently being developed, eliglustat tartrate (Genzyme Corporation), is hoped to have a better efficacy and toxicity profile, but, unfortunately, it cannot access the central nervous system. ${ }^{19}$ Thus, in the near future, five treatments - three enzymes and two substrate inhibitors - may become available that only treat the nonneuronopathic manifestations of Gaucher disease. As the majority of Gaucher patients have the non-neuronopathic phenotype, a broad arsenal of medication may still be important. This became clear during the dramatic shortage of imiglucerase (at the time the only authorized enzyme) that started in the summer of 2009 and continued, with ups and downs, for almost 2 years. Because many patients had to reduce or interrupt their therapy, there was a very welcome place for alternative enzymes. Although velaglucerase alfa has now been authorized, already providing a safe and effective alternative, many patients, especially those in non-Western countries, still suffer from untreated Gaucher disease. Obviously, this involves not only availability of enzymes but also, and more specifically, local reimbursement policies and facilities to treat and follow patients. In many countries, enzymes either cannot be administered, because appropriate knowledge and technical support is lacking, or cannot be reimbursed. Humanitarian programs, so far primarily supported by Genzyme Corporation, have stepped in to alleviate at least some of the need of these patients. This raises the point that cheaper medication may have a profound effect on access to treatment for more patients in other countries. The plant cell-derived system used for the manufacturing of taliglucerase alfa offers the possibility of large-scale production of the enzyme at lower cost. So far, the studies have shown that taliglucerase alfa is very effective, and this effectiveness also covers the extension period of the pivotal study, up to 24 months. Based upon the early reports, it seems that the safety-benefit ratio may be the same as for the two enzymes currently authorized. Perhaps an in-depth study on the immunologic effects, including acute hypersensitivity reactions, for the three enzymes should be performed, so as to identify risk factors that may aid in individualizing choice of therapy. In fact, to support individual treatment recommendations in the future, comparative studies of effectiveness and toxicity should be performed for all available therapies. Ideally, an independent committee should look for long-term outcomes for all treatments, as fragmentation of data in different industry-run registries hampers comparison of outcomes. ${ }^{20}$

\section{Conclusion}

Taliglucerase alfa has dose-dependent beneficial effects on the major manifestations of type I Gaucher disease. The drug is very well tolerated and has not led to any serious adverse events. Hypersensitivity reactions and antibody formation may occur, but it is unclear whether this is different from the two enzymes currently authorized. Taliglucerase alfa would be a very welcome addition to the treatment arsenal for Gaucher disease, as having an additional enzyme replacement therapy would reduce the risk for worldwide shortages of authorized enzymes. Furthermore, having an additional enzyme replacement therapy may result in cost reduction, therefore increasing possibilities for the treatment of patients living in less fortunate parts of the world.

\section{Acknowledgments/disclosure}

The author has received reimbursement of expenses and honoraria for lectures and occasional consultancies on the management of lysosomal storage diseases, including Gaucher disease, from Protalix Biotherapeutics, Genzyme Corporation, Actelion Pharmaceuticals Switzerland, and Shire plc. All honoraria are donated to the Gaucher Stichting, a national foundation that supports research in the field of lysosomal storage disorders. The author has worked with the team at the Academic Medical Center in Amsterdam, The Netherlands, on the interpretation of bone imaging data from a pivotal study. ${ }^{15}$

\section{References}

1. Beutler E, Grabowski GA. Gaucher disease. In: Scriver CR, Beaudet AL, Sly WS, Valle D, editors. The Metabolic and Molecular Bases of Inherited Disease. Vol 3. 8th ed. New York, NY: McGraw-Hill; 2001:3635-3668.

2. Barton NW, Brady RO, Dambrosia JM, et al. Replacement therapy for inherited enzyme deficiency: macrophage-targeted glucocerebrosidase for Gaucher's disease. N Engl J Med. 1991;324(21):1464-1470.

3. Zimran A, Altarescu G, Philips M, et al. Phase 1/2 and extension study of velaglucerase alfa replacement therapy in adults with type 1 Gaucher disease: 48-month experience. Blood. 2010;115(23):4651-4656.

4. Opar A. 'Pharmers' hope for first plant drug harvest. Nat Rev Drug Discov. 2011;10(2):81-82. 
5. ShaaltielY, Bartfeld D, Hashmueli S, et al. Production of glucocerebrosidase with terminal mannose glycans for enzyme replacement therapy of Gaucher's disease using a plant cell system. Plant Biotechnol J. 2007;5(5):579-590.

6. Brumshtein B, Salinas P, Peterson B, et al. Characterization of geneactivated human acid-beta-glucosidase: crystal structure, glycan composition, and internalization into macrophages. Glycobiology. 2009;20(1):24-32.

7. Zimran A, Brill-Almon E, Chertkoff R, et al. Pivotal trial with plant cell-expressed recombinant glucocerebrosidase, taliglucerase alfa, a novel enzyme replacement therapy for Gaucher disease. Blood. 2011; 118(22):5767-5773

8. Pastores G, Fernhoff P, Jeffrey S, et al. Plant cell expressed recombinant glucocerebrosidase - taliglucerase alfa - as therapy for Gaucher disease in patients previously treated with imiglucerase [meeting abstract]. Mol Genet Metab, Volume 105, Issue 2, February 2012, Page S50.

9. Aviezer D, Brill-Almon E, Shaaltiel Y, et al. A plant-derived recombinant human glucocerebrosidase enzyme: a preclinical and phase I investigation. PLoS One. 2009;4(3):e4792.

10. Hollak CE, van Weely S, van Oers MH, Aerts JM. Marked elevation of plasma chitotriosidase activity: a novel hallmark of Gaucher disease. J Clin Invest. 1994;93(3):1288-1292.

11. Hughes D, Cappellini MD, Berger M, et al. Recommendations for the management of the haematological and onco-haematological aspects of Gaucher disease. Br J Haematol. 2007;138(6):676-686.

12. Zimran A, Heitner R, Mehta A, et al. Long term safety and efficacy data of taliglucerase alfa, a plant cell expressed recombinant glucocerebrosidase, in treatment of naïve Gaucher disease patients. Mol Genet Metab. 2012;105(2):S68
13. Protalix announces new clinical data on taliglucerase alfa to be presented at the WORLD lysosomal disease network symposium [press release]. Karmiel, Israel: Protalix Biotherapeutics; February 9, 2012. Available from: http://phx.corporate-ir.net/phoenix.zhtml?c=101161\&p=irolnewsArticle\&ID=1658750\&highlight $/$. Accessed March 8,2012.

14. Personal communication Dr. E Almon, 2012.

15. Maas M, Hollak CE, Akkerman EM, Aerts JM, Stoker J, Den Heeten GJ. Quantification of skeletal involvement in adults with type I Gaucher's disease: fat fraction measured by Dixon quantitative chemical shift imaging as a valid parameter. Am J Roentgenol. 2002;179(4):961-965.

16. Van Dussen L, Hollak CEM, Zimran A, et al. Long term bone marrow responses, as measured by Quantitative Chemical Shift Imaging (QCSI) MRI, following treatment with taliglucerase alfa in patients with type 1 Gaucher disease. Mol Genet Metab. 2012;105(2):S62-S63.

17. Hollak CE, vom Dahl S, Aerts JM, et al. Force majeure: therapeutic measures in response to restricted supply of imiglucerase (Cerezyme) for patients with Gaucher disease. Blood Cells Mol Dis. 2010;44(1): $41-47$.

18. Cox TM, Aerts JM, Andria G, et al. The role of the iminosugar N-butyldeoxynojirimycin (miglustat) in the management of type I (non-neuronopathic) Gaucher disease: a position statement. $J$ Inherit Metab Dis. 2003;26(6):513-526.

19. Lukina E, Watman N, Arreguin EA, et al. Improvement in hematological, visceral, and skeletal manifestations of Gaucher disease type 1 with oral eliglustat tartrate (Genz-112638) treatment: 2-year results of a phase 2 study. Blood. 2010;116(20):4095-4098.

20. Hollak CE, Aerts JM, Aymé S, Manuel J. Limitations of drug registries to evaluate orphan medicinal products for the treatment of lysosomal storage disorders. Orphanet J Rare Dis. 2011;6:16.
Core Evidence

\section{Publish your work in this journal}

Core Evidence is an international, peer-reviewed open-access journal evaluating the evidence underlying the potential place in therapy of drugs throughout their development lifecycle from preclinical to postlaunch. The focus of each review is to evaluate the case for a new drug or class in outcome terms in specific indications and patient groups.

\section{Dovepress}

The manuscript management system is completely online and includes a very quick and fair peer-review system, which is all easy to use. Visit http://www.dovepress.com/testimonials.php to read real quotes from published authors. 